Gastroenterologia. 1940;65:I-IV

\title{
Contents, Vol. 65, 1940
}

\section{INDEX}

Cocchi, U., Kardiospasmus und Ulcus pepticum oesophagi 341

Danon, L., Contribution à l'étude des malformations du gros intestin 356

Demole, M., Note sur le mécanisme du ballonnement épi-

gastrique 236

Dimítríu, C. C, vide Tudoranu, G.

Friedrich, L. von, Die neue und eriolgreiche Heilmethode der Lamblia intestinalis .

- Interessante Fremdkörperfälle in der Speiseröhre und

im Magen 172

Froehlich, A. und H. J. Scherer, Zur Kenntnis der viscera-

len Form der Besnier-Boeckschen Krankheit . . 36 Grasser, C. H., Zur Röntgentherapie des

Oesophagus-

carzinoms 214

Heinemann, W., vide Sagal, Z.

Wan, B., vide Kapp, H.

Jezler, A., Eiweißkost beim Sport (Ergebnis einer Enquête

bei hervorragenden Schweizer Sportsleuten) . .244

Kapp, H. und B. Wan, Über die Bedeutung der chronischen

Gastritis für die Entwicklung des Magenkrebses . 137

- $\quad$ und E. Stamherger, Zur Cytodiagnostik der Gastritis 229

Kleeberg, J., Acute Severe Gastro-enteritis as a Problem

of Circulation and Permeability 149

Langen, C. de, Therapy and pathogenesis of ulcers oE the stomach and duodenum. (A clinical geographical study.) 1

Lundbaek, K., Gastroscopically Verified Oedema of the

Stomach in a Case of Familiar Quincke's Disease . 129

Lyon, E., On the Relationship between Vitamin A Metabolism and Thyrogenic Osteoporosis and Arthrosis õl

Petre, G., vide Tudoranu, G. i

Sagal, Z. und W. Heinemann, Kritische Betrachtungen

zur Appendicitisfrage 80

Sauser-Hall, P., Pneumatosis cystoïdes intestini . 193, 313

Scherer, H. J., vide Froehlich, A.

Schmid, E. A., Purgatio partialis et generalis, mit erwarteten und unerwarteten Heilwirkungen im Stoff-und Kraftwechsel durch subaquale Darmbäder und kombinierte Diät 258 
Stamberger, E., vide Kapp.

Sucher, A., Fortschritte in der Behandlung des kompletten

Gallenversehlusses mittels Duodenalsondierungen . 162

Tanasoca, T., vide Yudoranu, G.

Tudoranu, G., C. C. Dimítríu, T. Tanasoca und G. Petre, Über den Einfluß der Absorption durch den Zwölí-

fingerdarm auf die Motrizität des Magens beimMen-

schen (Viszerographie) 72

Wallbach, G., Examens morphologiques concernant I'aetion

des remèdes sécrétoires sur les inflammations in-

testinales 63

Casuistique - Kasuistik - Case Reports

Feißly, R., Un cas de polype solitaire du colon descen

dant 273

Un cas de sténose du cardia par cicatrice d'ulcus . 277

Un cas de hernie au travers du hiatus oesophagien . 278 Stalder, H., Die Wirkung des

Corticosterons bei der idio-

pathischen Steatorrhoe

280

Therapia 101

Referate - Abstracts - Analyses . . 102, 179, 284, 372

Max Askanazy $\dagger \quad 306$

Buchbesprechungen - Books Review - Livres Nou-

veaux 128,308 